\title{
Digital Library Programs for Libraries and Archives: Developing, Managing, and
} Sustaining Unique Digital Collections. By Aaron D. Purcell. Chicago: ALA NealSchuman, 2016. 256 pp. Index. Softcover. $\$ 85.00$.

Aaron Purcell's Digital Library Programs offers a broad overview of the current landscape of digital library and archives work and provides guidance on establishing digital projects and more permanent digital programs. This book was written as a result of his experiences working in a digital archives context and developing and teaching several courses in digital libraries. The early chapters in part 1, "The Theory and Reality of Digital Libraries," situate digitization in the wider world of library and archives services and are good primers for those who are new to the field. This book really shines, however, in part 2, "Building Digital Library Programs: A Step-by-Step Process," where it discusses ways to think about specific issues surrounding the establishment of a digitization program. A book like this poses a significant problem for its author: any concrete advice offered about which products to purchase is likely outdated by the time the book goes to press. In addition, each institution has its own priorities, funding structure, staffing situation, and unique collections, so blanket advice about how to develop a digitization program may not be particularly helpful. Purcell gets around this problem by identifying key issues involved in building a digitization program and by providing exercises at the end of each chapter that build upon suggestions in the chapter, raise additional points, and enable readers to address these issues in their own institutional contexts. Part 3, "Digital Library Planning Exercises," features additional exercises that help readers synthesize the information in each chapter and apply it to their own institutions. So, while readers will not finish this book with a concrete list of what software and systems to set up, they will begin to conceptualize which questions to ask and how to determine what products will meet the needs of their projects and institutions.

The book begins with a brief history of electronic records and computing and their role in the library world. Similarly, chapter 2, "Context of Today's Libraries and Digital Libraries," describes the role digital materials fill in a world where libraries face decreased funding, space limitations, and demands to support ever-changing technologies. These chapters attempt to situate the ensuing narrative in a particular context, but the intended audience is unclear. While the rest of the book feels like a handbook for guiding practitioners through implementing a digital library program, these first two chapters seem to be written for readers who are unfamiliar with the world of contemporary librarianship or those interested in the history of computing in librarianship.

In chapters 3 and 4, Purcell begins to narrow his focus to digitization projects. Chapter 3, "Digitization and Digital Libraries," discusses the process of preparing for the digitization of library materials, including deciding what to digitize, why to digitize, and how to start conceiving digitization projects. Chapter 4, "Vision and Mission Building," outlines ways to build vision and mission statements and describes how these can provide strong guidelines for keeping projects on track. Chapter 5, "Identifying Resources and Partnerships," is particularly strong, as it takes a broad view of the staff required to create and maintain a digital program, including less visible staff such as technical personnel. This chapter also discusses ways to customize each digitization program to 
the available resources. Purcell wisely reminds readers that " $\mathrm{t}]$ he point of digitization is not to transform a small public library with three staff into a large-scale digital production facility. Instead, each library has the potential to contribute to the larger purpose of providing free online access to original or rare materials of historical significance" (p. 77). As a practitioner, it is easy to feel that only those institutions rich in resources and staffing expertise can establish a digitization program the "right way," but Purcell is able to help readers from institutions of all sizes and resource levels to identify ways to build a program within their means.

In chapter 6, "Evaluating, Selecting, and Building Digital Collections," Purcell outlines the various types of primary source materials that libraries and archives might consider digitizing and describes format challenges and strengths for each. This section will be of particular interest to librarians who do not routinely work with nonprint or unpublished formats. Purcell also provides a good overview of copyright issues in this chapter. In chapter 7, “Technical Standards,” Purcell tackles digitization workflows, product selection, and technical specifications. Because of the broad scope of this book, he is unable to go into great detail about which products to select and why. In fact, this chapter is the same length as the other chapters, although technical issues are often the first concerns practitioners have when establishing a digitization program. Purcell wisely touches on the basics while providing guidance on how each institution should go about establishing specifications that suit its particular circumstances.

Chapters 8 through 10, on "Management of Digital Projects, "Outreach and Instruction," and "Promotion, Assessment, and Sustainability," describe ways in which traditional processes in archives and library work apply to digital projects. For example, in chapter 9, Purcell provides a nice overview of the many ways to think about access in the context of digital projects. He mentions public access to digital content via the Internet; information security relating to internal access to a digital project; choosing a public user interface for navigating digital content; creating access points and tools; metadata; and considering what features the intended audience may desire in regard to the digital content. In chapter 11, "Planning Digital Library Programs," Purcell addresses ways to turn one-off digital projects into more permanent digital programs, touching on ways to balance speed, quality, and costs. I do wish Purcell had spent more time discussing long-term digital preservation, as that is often a huge, expensive, and onerous part of digital project planning.

This book would be most useful for practitioners who are new to digital projects and would like a broad outline of issues to consider as they embark on establishing a program. However, it also provides useful tips for practitioners who have worked in digital initiatives for a while. For example, the sections on assessment, sustainability, and longrange planning would benefit digital project leaders who are midway through projects and beginning to address longer-term issues.

Jessica Wagner Webster

Digital Initiatives Librarian

Baruch College, City University of New York 